\title{
Patients' satisfaction with medication information provided by nurses using independent nurse prescribing (INP) or patient group directions (PGDs) in UK sexual health services.
}

\author{
Black, A.'; Courtenay, M..; Gage, H.; Norton, C.1; Franklin, B.D. ${ }^{4}$
}

${ }^{1}$ King's College London; ${ }^{2}$ Cardiff University; ${ }^{3}$ University of Surrey; ${ }^{4}$ Imperial College Healthcare NHS Trust
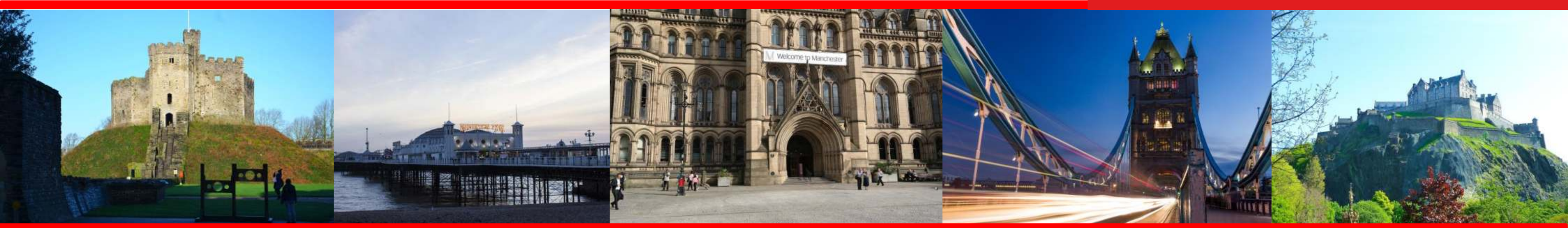

\section{INTRODUCTION}

Nurses' ability to independently deliver medication was introduced to improve patients' access to medication and improve experience of healthcare services ${ }^{[1-2]}$. Independent nurse prescribing (INP) and patient group directions (PGDs) are two methods that allow nurses to independently deliver medications ${ }^{[3-4]}$. Both methods are frequently used in sexual health services ${ }^{[5]}$; however, there is limited evidence of patients' satisfaction with medication information provided. This study set out to explore that gap.

\section{RESULTS}

Of the 393 patients who received a questionnaire, 92\% ( $n=360)$ responded. Patients' feedback for INP and PGD nurses was so similar that aggregate data are presented.

Patients'satisfaction with medication consultations

Patients clearly identified that nurses managing their medication consultations were:

- Friendly and approachable

- Instilled confidence and trust

- Explained the reasons for medications clearly

- Suitably answered questions

\begin{abstract}
METHODS
Nurses (INP or using PGD) from five UK city based sexual health services distributed a questionnaire survey to patients with whom they had consulted and delivered medication, Sept 2015 - Aug 2016. The questionnaire was informed by Birmingham's sexual health service satisfaction questionnaire and the Satisfaction with Information about Medicines Scale (SIMS). SIMS classifies: 'about right' and 'not applicable' as satisfied responses, whereas 'too much', 'too little', 'none received' as unsatisfied.
\end{abstract}

Patients' satisfaction with nursing consultations

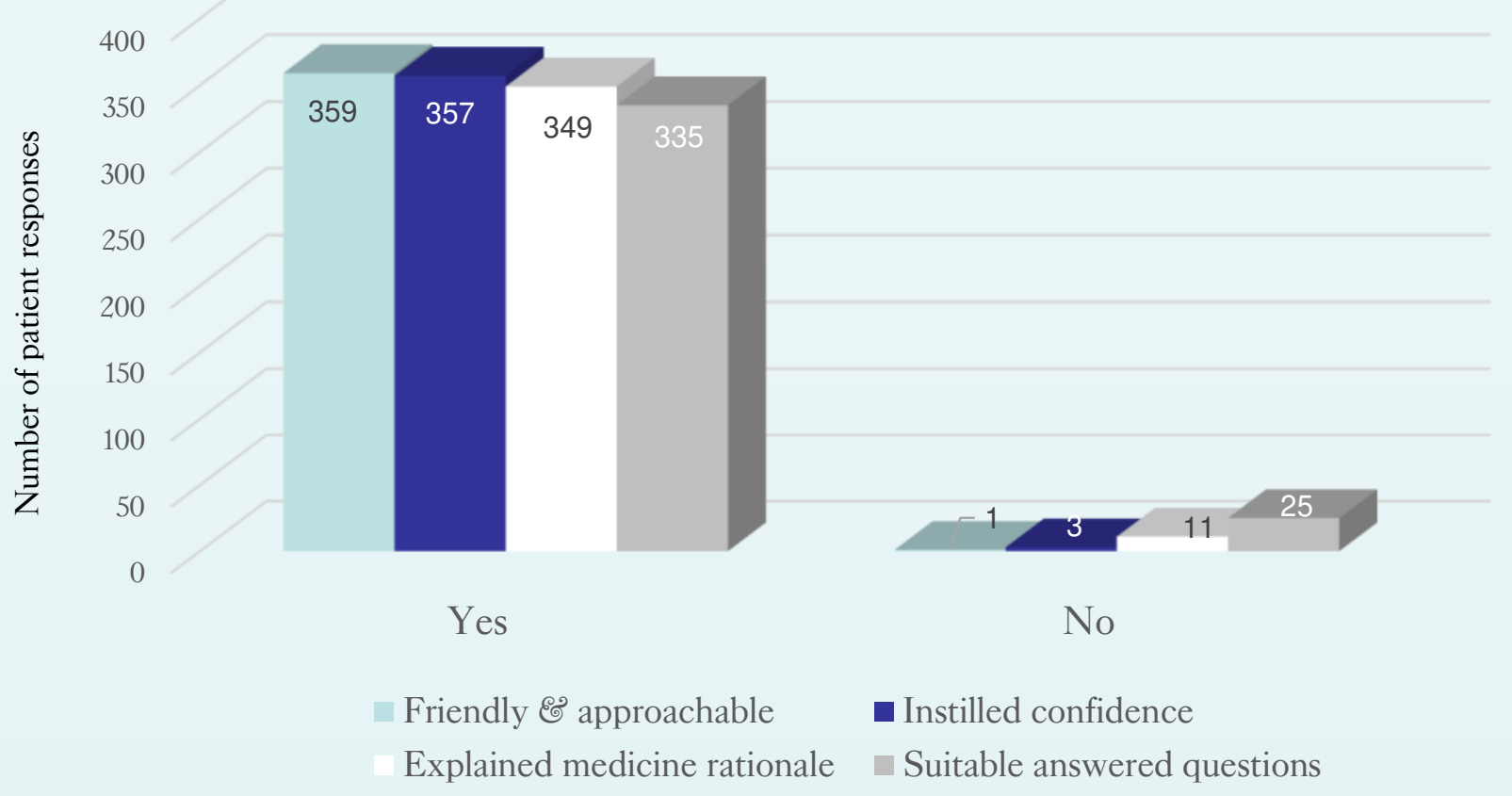

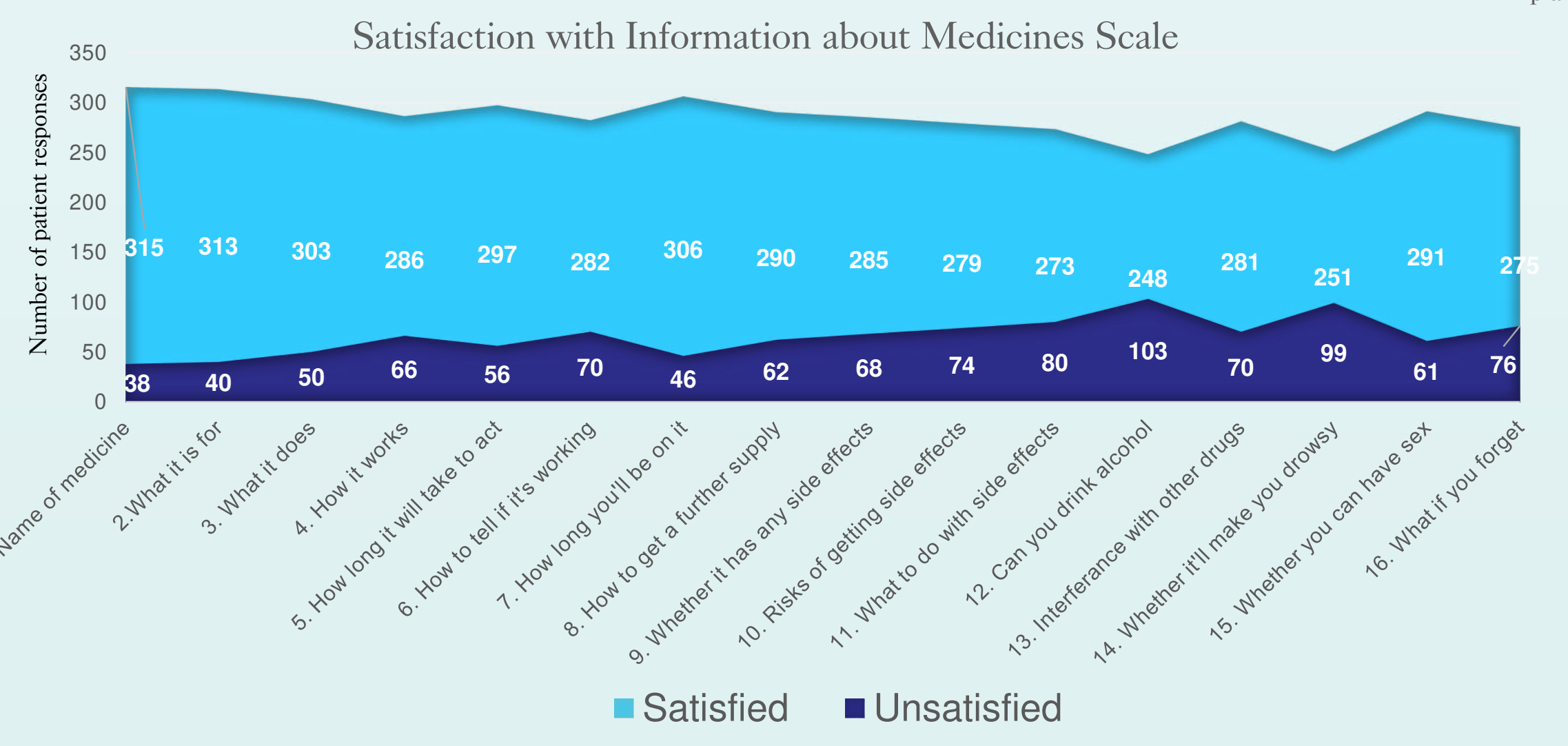

SIMS

Of the $89 \%(n=348 / 360)$ of respondents who completed the SIMS, an overall score of 13.3/16 was achieved: the higher the score, the greater the satisfaction. The largest points of dissatisfaction related to not receiving satisfactory information on:

- Whether they could drink alcohol

- Potential for drowsiness

- How to manage side effects

\section{DISCUSSION}

Patients predominantly provided very positive feedback regarding their medication consultations with nurses. There was extremely high satisfaction with INP/ PGD nurses' consultation skills; although a small proportion of patients indicated they would have liked more opportunities to ask questions. High SIMS scores identified overall satisfaction with medication information; however, more consideration may be needed on the potential problems medications can cause (e.g. advice on alcohol consumption, side effects and drowsiness potential). However, the majority of medicines used in sexual health are unlikely to be affected by alcohol or cause drowsiness ${ }^{[6-7]}$, therefore, this may not be essential advice in all cases. 